\title{
On a Class of Isotopic Connectivity of Gradient-like Maps of the 2-sphere with Saddles of Negative Orientation Type
}

\author{
T. V.Medvedev, E. V.Nozdrinova, O. V.Pochinka, E. V.Shadrina
}

We consider the class $G$ of gradient-like orientation-preserving diffeomorphisms of the 2sphere with saddles of negative orientation type. We show that the for every diffeomorphism $f \in G$ every saddle point is fixed. We show that there are exactly three equivalence classes (up to topological conjugacy) $G=G_{1} \cup G_{2} \cup G_{3}$ where a diffeomorphism $f_{1} \in G_{1}$ has exactly one saddle and three nodes (one fixed source and two periodic sinks); a diffeomorphism $f_{2} \in G_{2}$ has exactly two saddles and four nodes (two periodic sources and two periodic sinks) and a diffeomorphism $f_{3} \in G_{3}$ is topologically conjugate to a diffeomorphism $f_{1}^{-1}$. The main result is the proof that every diffeomorphism $f \in G$ can be connected to the "source-sink" diffeomorphism by a stable arc and this arc contains at most finitely many points of period-doubling bifurcations.

Keywords: sink-source map, stable arc

Received June 05, 2019

Accepted June 20， 2019

The construction of a stable arc (Theorem 2) is supported by RSF (Grant no. 17-11-01041), the splitting $G$ into equivalence classes (Theorem 1) is supported by the Basic Research Program at the National Research University Higher School of Economics (HSE) in 2019.

Timur V. Medvedev

tmedvedev@hse.ru

National Research University Higher School of Economics

ul. Rodionova 136, Niznhy Novgorod, 603093 Russia

Elena V. Nozdrinova

maati@mail.ru

Olga V. Pochinka

olga-pochinka@yandex.ru

Elena V. Shadrina

eshadrina@hse.ru

National Research University Higher School of Economics

ul. Bolshaya Pecherckaya 25/12, Niznhy Novgorod, 603155 Russia 


\section{Introduction}

In 1976 S. Newhouse, J. Palis and F. Takens [1] introduced the notion of a stable arc connecting two structurally stable systems on a manifold, the arc being stable in the sense that any nearby arc exhibits the same type of behavior. Soon S. Newhouse and M. Peixoto [2] showed the existence of a stable arc between any two Morse-Smale flows. On the other hand, there are examples of Morse-Smale diffeomorphisms on manifolds of any dimension that cannot be joined by a stable arc. Therefore, it is important to find an invariant which defines the equivalence class of Morse-Smale diffeomorphisms with respect to relation of connectivity by a stable arc (stable connectivity component).

For orientation-preserving structurally stable maps of the circle the Poincaré rotation number is this invariant [3]. P. Blachard found [4] some necessary conditions for existence of a stable arc connecting two Morse-Smale diffeomorphisms on surfaces. These conditions, in particular, imply that even on the 2 -sphere there are infinitely many stable connectivity components. The simplest example of a 2-dimensional diffeomorphism is the "source-sink" system on the 2-sphere. The main result of this paper is the proof that a gradient-like orientation-preserving diffeomorphism of the 2-sphere with saddles of negative orientation type can be joined by a stable arc to the "source-sink" diffeomorphism.

\section{Dynamics of $G$ diffeomorphisms}

Let $M^{n}$ be a smooth closed orientable manifold with a metric $d$ and let $f: M^{n} \rightarrow M^{n}$ be an orientation-preserving diffeomorphism. A point $x \in M^{n}$ is said to be wandering for $f$ if there is an open neighborhood $U_{x}$ of $x$ such that $f^{n}\left(U_{x}\right) \cap U_{x}=\emptyset$ for all $n \in \mathbb{N}$. Otherwise the point $x$ is called nonwandering. The set of all nonwandering points of $f$ is called the nonwandering set, denoted by $\Omega_{f}$.

If $\Omega_{f}$ is finite, then each $p \in \Omega_{f}$ is periodic and its period $m_{p} \in \mathbb{N}$. A point $p \in \Omega_{f}$ is called hyperbolic if the Jacobian matrix $\left.\left(\frac{\partial f^{m_{p}}}{\partial x}\right)\right|_{p}$ has no eigenvalues on the unit circle. If all the eigenvalues lie inside (outside) the unit circle, then $p$ is a sink (a source). Sinks and sources are called nodes. If a hyperbolic periodic point is not a node, then it it called a saddle.

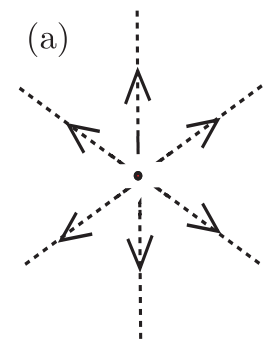

source

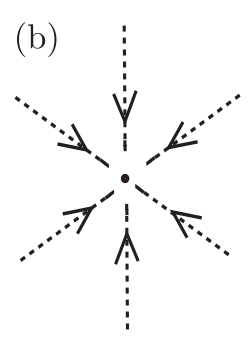

$\operatorname{sink}$

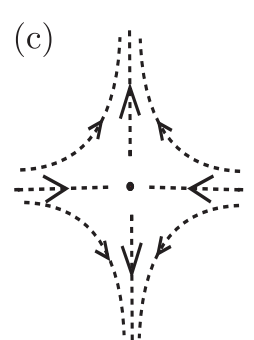

saddle

Fig. 1. (a) source, (b) sink, (c) saddle. 
The hyperbolic structure of a periodic point $p$ implies the existence of the stable $W_{p}^{s}$ and the unstable $W_{p}^{u}$ manifolds defined by

$$
\begin{aligned}
& W_{p}^{s}=\left\{x \in S_{g}: \lim _{k \rightarrow+\infty} d\left(f^{k \cdot p \operatorname{per}(p)}(x), p\right)=0\right\}, \\
& W_{p}^{u}=\left\{x \in S_{g}: \lim _{k \rightarrow+\infty} d\left(f^{-k \cdot \operatorname{per}(p)}(x), p\right)=0\right\} ;
\end{aligned}
$$

$W_{p}^{s}$ and $W_{p}^{u}$ are diffeomorphic to $\mathbb{R}^{n-q_{p}}, \mathbb{R}^{q_{p}}$, respectively, where $q_{p}$ is the number of the eigenvalues of the Jacobian matrix outside the unit circle.

Stable and unstable manifolds are called invariant manifolds. A path-component of the set $W_{p}^{u} \backslash p\left(W_{p}^{s} \backslash p\right)$ is called an unstable (stable) separatrix.

Periodic data of the periodic orbit $\mathcal{O}_{p}$ of a periodic point $p$ is the collection $\left(m_{p}, q_{p}, \nu_{p}\right)$ where $m_{p}$ is the period of $p, q_{p}=\operatorname{dim} W_{p}^{u}$ and $\nu_{p}$ is the type of orientation, meaning $p: p=+1(p=-1)$ if $\left.f^{m_{p}}\right|_{W_{p}^{u}}$ preserves (reverses) the orientation. For orientation-preserving diffeomorphisms the orientation type of each node is +1 , whereas the orientation type of a saddle can be +1 as well as -1 .

A diffeomorphism $f: M^{n} \rightarrow M^{n}$ is called a Morse-Smale diffeomorphism if

1) the nonwandering set $\Omega_{f}$ consists of a finite number of hyperbolic orbits;

2) the manifolds $W_{p}^{s}, W_{q}^{u}$ of each two distinct nonwandering points $p$ and $q$ intersect transversally.

A Morse-Smale diffeomorphism is called gradient-like if from $W_{\sigma_{1}}^{s} \cap W_{\sigma_{2}}^{u} \neq \emptyset$ for two distinct points $\sigma_{1}, \sigma_{2} \in \Omega_{f}$ it follows that $\operatorname{dim} W_{\sigma_{1}}^{u}<\operatorname{dim} W_{\sigma_{2}}^{u}$.

A Morse-Smale flow on a manifold $M^{n}$ is defined in the same way. A Morse-Smale flow is said to be gradient-like if it has no periodic trajectories.

For $n=2$ the dynamics of gradient-like diffeomorphisms is closely interconnected with the dynamics of periodic homeomorphisms. Recall that a homeomorphism $\phi: M^{2} \rightarrow M^{2}$ is periodic of order $m \in \mathbb{N}$ if $\phi^{m}=i d$ and $\phi^{\mu} \neq i d$ for each natural $\mu<m$.

Proposition 1 ([5], Theorem 3.3). Every orientation-preserving gradient-like diffeomorphism $f: M^{2} \rightarrow M^{2}$ is topologically conjugate to the composition of a periodic homeomorphism and the time-1 map of a gradient-like flow.

According to the classification by B. von Kerekjarto [6], periodic points of an orientationpreserving periodic homeomorphism of period $m$ on the 2-sphere can only be of periods 1 and $m$, whereas the set of fixed points of this diffeomorphism is not empty. Hence, he have the following corollary of Proposition 1.

Proposition 2. Periodic points of an orientation-preserving gradient-like diffeomorphism of the 2-sphere can only be of period 1 or of period $m$ (the case $m=1$ is possible). The set of fixed points of this diffeomorphism is not empty.

For an orientation-preserving gradient-like diffeomorphism $f: M^{2} \rightarrow M^{2}$ the following proposition is also true.

Proposition 3 ([5], Lemmas 3.1, 3.3). Let $f: M^{2} \rightarrow M^{2}$ be an orientation-preserving gradient-like diffeomorphism and let $m_{f}$ be the least natural for which $\Omega_{f^{m_{f}}}$ consists of the fixed points of positive orientation type. Then the period of each saddle separatrix of $f$ equals $m_{f}$.

Thus, from Propositions 2 and 3 we have 
Proposition 4. Let $f$ be an orientation-preserving gradient-like diffeomorphism of the 2-sphere. Then

1) $m_{f}=m$;

2) each saddle with negative orientation type is fixed.

Recall that the 2-sphere is a manifold diffeomorphic to $\mathbb{S}^{2}=\left\{\left(x_{1}, x_{2}, x_{3}\right) \in \mathbb{R}^{3}: x_{1}^{2}+x_{2}^{2}+\right.$ $\left.+x_{3}^{2}=1\right\}$. Denote by $G$ the set of gradient-like orientation-preserving diffeomorphisms of the 2-sphere with saddles of negative orientation type.

In this paper we give a topological classification of diffeomorphisms of $G$, namely, we prove the following theorem.

Theorem 1. There are three equivalence classes $G=G_{1} \cup G_{2} \cup G_{3}$ up to topological conjugacy, where a diffeomorphism $f_{1} \in G_{1}$ has exactly one saddle and three nodes: one fixed source and two periodic sinks (Fig. 2); a diffeomorphism $f_{2} \in G_{2}$ has exactly two saddles and four nodes: two periodic sources and two periodic sinks (Fig. 3); a diffeomorphism $f_{3} \in G_{3}$ is topologically conjugate to a diffeomorphism $f_{1}^{-1}$ (Fig. 4).

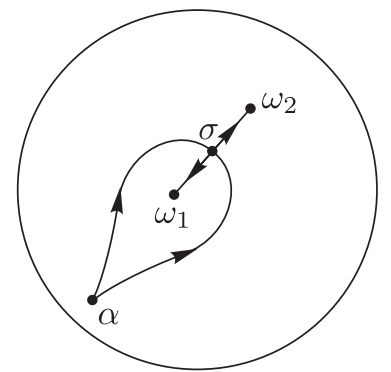

Fig. 2. $f_{1} \in G_{1}$.

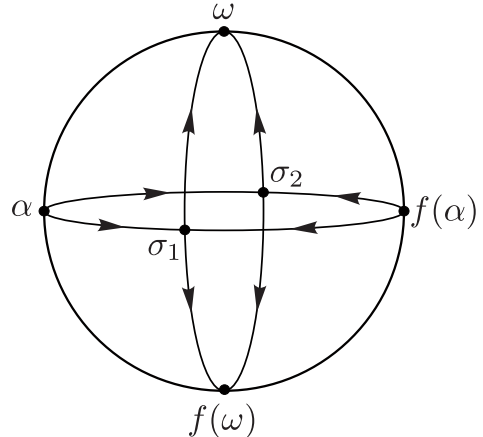

Fig. 3. $f_{2} \in G_{2}$.

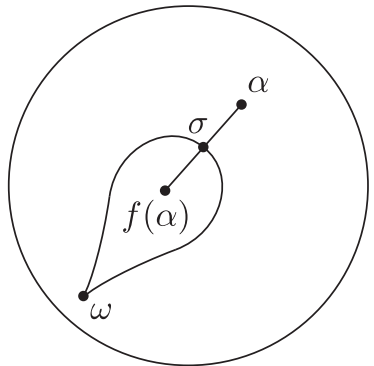

Fig. 4. $f_{3} \in G_{3}$.

\section{Stable arcs in the space of diffeomorphisms}

Consider a 1-parametric family of diffeomorphisms (an arc) $\varphi_{t}: M^{n} \rightarrow M^{n}, t \in[0,1]$. Denote by $\mathcal{Q}$ the set of arcs $\left\{\varphi_{t}\right\}$ whose end points are Morse-Smale diffeomorphisms and such that:

1) every diffeomorphism $\varphi_{t}$ has a finite limit set for every $t \in[0,1]$;

2) the arc $\left\{\varphi_{t}\right\}$ contains a finite set of bifurcation points $b_{1}, \ldots, b_{m} \in(0,1)$.

Following [7], an arc $\left\{\varphi_{t}\right\}$ is called stable if it is an inner point of the equivalence class with respect to the following relation: two $\operatorname{arcs}\left\{\varphi_{t}\right\},\left\{\varphi_{t}^{\prime}\right\} \in \mathcal{Q}$ are called conjugate if there are homeomorphisms $h:[0,1] \rightarrow[0,1], H_{t}: M^{n} \rightarrow M^{n}$ such that $h\left(b_{i}\right)=b_{i}^{\prime}, i \in\{1, \ldots, m\}$, $H_{t} \varphi_{t}=\varphi_{h(t)}^{\prime} H_{t}, t \in[0,1]$ and $H_{t}$ continuously depend on $t$.

It is shown in [7] that an $\operatorname{arc}\left\{\varphi_{t}\right\} \in \mathcal{Q}$ is stable if and only if 
- each diffeomorphism $\varphi_{b_{i}}, i \in\{1, \ldots, m\}$ has no cycles and it has exactly one nonhyperbolic periodic orbit, this orbit being a flip or noncritical saddle-node, and the arc passes through the bifurcation point in the typical way;

- stable and unstable manifolds of any two periodic points of the diffeomorphism $\varphi_{t}, t \in[0,1]$ intersect transversally (Fig. 5).

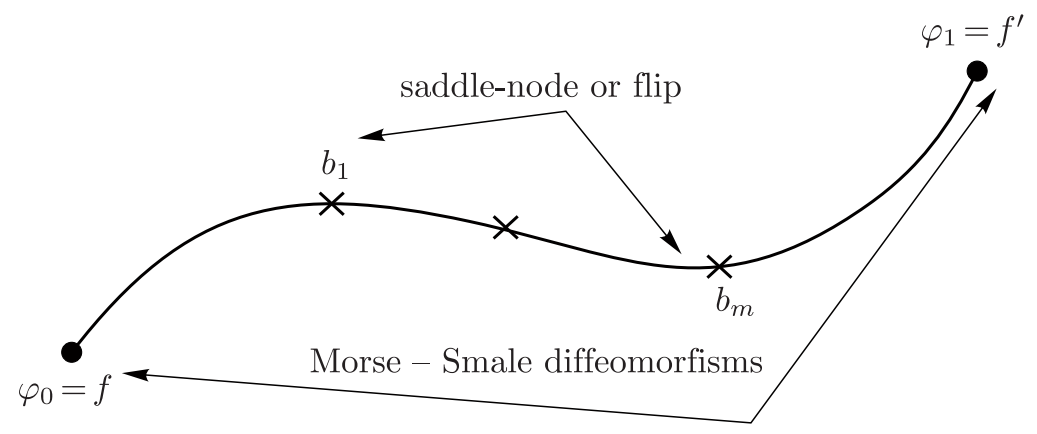

Fig. 5. An arc from $\mathcal{Q}$.

We say that two diffeomorphisms $f_{0}, f_{1}$ are in the same class of stable isotopic connectivity if they can be joined in the space of diffeomorphisms by an arc of $\mathcal{Q}$.

An $\operatorname{arc}\left\{\varphi_{t}\right\} \in \mathcal{Q}$ is said to pass through a saddle-node bifurcation $\varphi_{b_{i}}$ in the typical way if in some neighborhood of the nonhyperbolic point $\left(p, b_{i}\right)$ the $\operatorname{arc} \varphi_{t}$ is conjugate to

$$
\begin{gathered}
\tilde{\varphi}_{\tilde{t}}\left(x_{1}, x_{2}, \ldots, x_{1+n_{u}}, x_{2+n_{u}}, \ldots, x_{n}\right)= \\
\left(x_{1}+\frac{1}{2} x_{1}^{2}+\tilde{t}, \pm 2 x_{2}, \ldots, \pm 2 x_{1+n_{u}}, \frac{ \pm x_{2+n_{u}}}{2}, \ldots, \frac{ \pm x_{n}}{2}\right),
\end{gathered}
$$

where $\left(x_{1}, \ldots, x_{n}\right) \in \mathbb{R}^{n},\left|x_{i}\right|<1 / 2,|\tilde{t}|<1 / 10$.
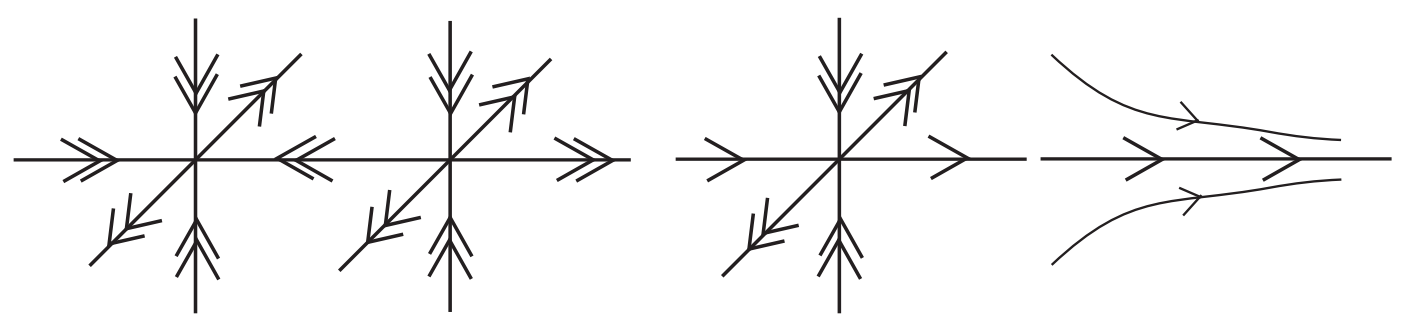

Fig. 6. Saddle-node.

An $\operatorname{arc}\left\{\varphi_{t}\right\} \in \mathcal{Q}$ is said to pass through period-doubling bifurcation (flip) $\varphi_{b_{i}}$ in the typical way if in some neighborhood of the nonhyperbolic point $\left(p, b_{i}\right)$ the $\operatorname{arc} \varphi_{t}$ is conjugate to the arc

$$
\begin{gathered}
\tilde{\varphi}_{\tilde{t}}\left(x_{1}, x_{2}, \ldots, x_{1+n_{u}}, x_{2+n_{u}}, \ldots, x_{n}\right)= \\
\left(-x_{1}(1 \pm \tilde{t})+x_{1}^{3}, \pm 2 x_{2}, \ldots, \pm 2 x_{1+n_{u}}, \frac{ \pm x_{2+n_{u}}}{2}, \ldots, \frac{ \pm x_{n}}{2}\right),
\end{gathered}
$$

where $\left(x_{1}, \ldots, x_{n}\right) \in \mathbb{R}^{n},\left|x_{i}\right|<1 / 2,|\tilde{t}|<1 / 10$.

The main result of this paper is the following theorem.

Theorem 2. Every diffeomorphism $f \in G$ is connected to the "source-sink" diffeomorphism by a stable arc. 

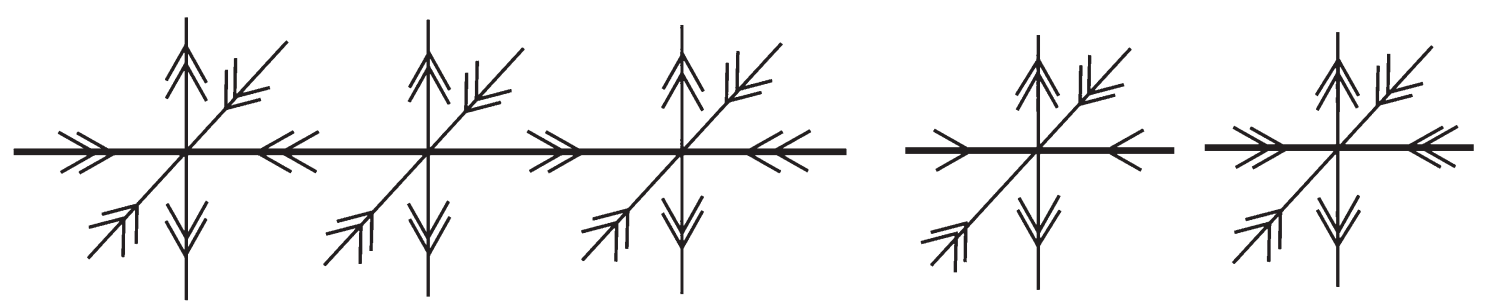

Fig. 7. A period-doubling bifurcation (flip).

\section{Proof of Theorem 1}

In this section we prove that up to topological conjugacy there are exactly three equivalence classes $G=G_{1} \cup G_{2} \cup G_{3}$ where a diffeomorphism $f_{1} \in G_{1}$ has exactly one saddle and three nodes - one fixed source and two periodic sinks; a diffeomorphism $f_{2} \in G_{2}$ has exactly two saddles and four nodes - two periodic sources and two periodic sinks (Fig. 3); a diffeomorphism $f_{3} \in G_{3}$ is topologically conjugate to a diffeomorphism $f_{1}^{-1}$ (Fig. 4).

\section{Proof.}

Let $f \in G$. It follows from the properties of $G$ that any saddle $\sigma$ of $f$ is of negative orientation type. Then, according to Proposition 4 , the point $\sigma$ is fixed. Denote by $\ell_{\sigma}^{1}, \ell_{\sigma}^{2}$ the stable separatrices of $\sigma$ and denote by $\gamma_{\sigma}^{1}, \gamma_{\sigma}^{2}$ the unstable separatrices of $\sigma$. Consider two cases

1) there is a saddle $\sigma$ such that $\operatorname{cl}\left(\ell_{\sigma}^{1}\right) \backslash \ell_{\sigma}^{1}=\operatorname{cl}\left(\ell_{\sigma}^{2}\right) \backslash \ell_{\sigma}^{2}$ or $\operatorname{cl}\left(\gamma_{\sigma}^{1}\right) \backslash \gamma_{\sigma}^{1}=\operatorname{cl}\left(\gamma_{\sigma}^{2}\right) \backslash \gamma_{\sigma}^{2}$;

2) there are no saddles satisfying 1 ).

In the case 1) to be definite consider $\operatorname{cl}\left(\ell_{\sigma}^{1}\right) \backslash \ell_{\sigma}^{1}=\operatorname{cl}\left(\ell_{\sigma}^{2}\right) \backslash \ell_{\sigma}^{2}$ (Fig. 2). Since $f$ is gradient-like, the set $\operatorname{cl}\left(\ell_{\sigma}^{1}\right) \backslash \ell_{\sigma}^{1}=\operatorname{cl}\left(\ell_{\sigma}^{2}\right) \backslash \ell_{\sigma}^{2}$ is the source; denote it by $\alpha$. Thus,

$$
\alpha=\operatorname{cl}\left(\ell_{\sigma}^{1}\right) \backslash \ell_{\sigma}^{1}=\operatorname{cl}\left(\ell_{\sigma}^{2}\right) \backslash \ell_{\sigma}^{2}
$$

and then the set $K=\alpha \cup \ell_{\sigma}^{1} \cup \ell_{\sigma}^{2} \cup \sigma$ is the circle which is invariant under $f$. On the ambient 2-sphere this circle is the border of two 2-disks $D_{1}, D_{2}$ such that $\gamma_{\sigma}^{1} \subset D_{1}, \gamma_{\sigma}^{2} \subset D_{2}$. Since $\sigma$ is of negative orientation type, we have $f\left(D_{1}\right)=D_{2}, f\left(D_{2}\right)=D_{1}$.

Hence, the disk $D_{1}$ is of period two and, consequently, cannot contain saddle points because they should be fixed. The Euler characteristic of the 2-sphere equals 2, therefore, there are exactly two sinks $\omega_{1} \in D_{1}, \omega_{2} \in D_{2}$ lying in the closures of the unstable separatrices $\gamma_{\sigma}^{1}, \gamma_{\sigma}^{2}$, respectively, and such that $f\left(\omega_{1}\right)=\omega_{2}, f\left(\omega_{2}\right)=\omega_{1}$.

So, in this case the nonwandering set of $f$ is $\Omega_{f}=\{\alpha, \omega, f(\omega), \sigma\}$. Having applied the same reasoning for the case $\operatorname{cl}\left(\gamma_{\sigma}^{1}\right) \backslash \gamma_{\sigma}^{1}=\operatorname{cl}\left(\gamma_{\sigma}^{2}\right) \backslash \gamma_{\sigma}^{2}$, we come to a diffeomorphism conjugate to $f^{-1}$.

Consider case 2). Let $\sigma_{1}$ be a saddle of $f$. Then all the four saddle separatrices have four distinct nodes in their closures. Let $c l\left(\ell_{\sigma_{1}}^{1}\right) \backslash \ell_{\sigma_{1}}^{1}=\alpha, \operatorname{cl}\left(\ell_{\sigma_{1}}^{2}\right) \backslash \ell_{\sigma_{1}}^{2}=f(\alpha)$ and $\operatorname{cl}\left(\gamma_{\sigma_{1}}^{1}\right) \backslash \gamma_{\sigma_{1}}^{1}=$ $=\omega, c l\left(\gamma_{\sigma_{1}}^{2}\right) \backslash \gamma_{\sigma_{1}}^{2}=f(\omega)$ (Fig. 8).

Since the Euler characteristic of the 2-sphere equals 2, the nonwandering set of $f$ contains at least one saddle distinct from $\sigma_{1}$. Since the set $\mathbb{S}^{2} \backslash P$ is a connected manifold (here $\mathbb{S}^{2}$ is the 2 -sphere and $P$ is the set of sources of $f$ ), the union of the unstable manifolds of all saddles is connected. Therefore, there exists a saddle $\sigma_{2}$ such that

$$
\operatorname{cl}\left(\gamma_{\sigma_{2}}^{1}\right) \backslash \gamma_{\sigma_{2}}^{1}=\operatorname{cl}\left(\gamma_{\sigma_{1}}^{1}\right) \backslash \gamma_{\sigma_{1}}^{1}=\omega .
$$




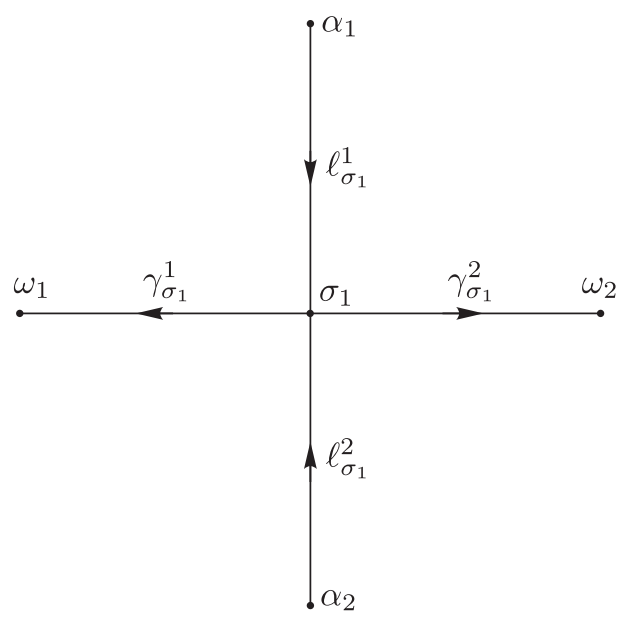

Fig. 8. The saddle $\sigma_{1}$.

Since the saddles are of negative orientation type, we have $f\left(\gamma_{\sigma_{2}}^{1}\right)=\gamma_{\sigma_{2}}^{2}$ and

$$
\operatorname{cl}\left(\gamma_{\sigma_{2}}^{2}\right) \backslash \gamma_{\sigma_{2}}^{2}=\operatorname{cl}\left(\gamma_{\sigma_{1}}^{2}\right) \backslash \gamma_{\sigma_{1}}^{2}=f(\omega)
$$

Thus, the set $K=\omega \cup f(\omega) \cup \gamma_{\sigma_{1}}^{1} \cup \gamma_{\sigma_{1}}^{2} \cup \gamma_{\sigma_{2}}^{1} \cup \gamma_{\sigma_{2}}^{2}$ is an $f$-invariant circle. By the same reasoning as in case 1 ) we find that there are no saddles of $f$ distinct from $\sigma_{1}, \sigma_{2}$ and, hence, $\Omega_{f}=\left\{\sigma_{1}, \sigma_{2}, \omega, f(\omega), \alpha, f(\alpha)\right\}$.

\section{Reduction of diffeomorphisms to the standard form}

To decrease the number of periodic orbits of a diffeomorphism $f \in G$, we are going to construct an arc that passes through a period-doubling bifurcation in the typical way. In order to do this, the diffeomorphisms must be reduced to the standard form. To that end we reduce the dynamics in the neighborhood of a sink to the canonical contraction (Lemma1) and we map the unstable saddle separatrix in the basin of the canonical sink to a smooth arc (Lemma 2). An important tool for all the constructions is

Proposition 5 (Thom's isotopy extension theorem, [8], Theorem 5.8). Let $Y$ be a smooth manifold without border, let $X$ be a smooth submanifold of $Y$ and let $\left\{f_{t}: X \rightarrow Y, t \in\right.$ $[0,1]\}$ be a smooth isotopy such that $f_{0}$ is the inclusion map of $X$ into $Y$. Then for every compact set $A \subset Y$ containing the support ${ }^{1} \operatorname{supp}\left\{f_{t}\right\}$ there is a smooth isotopy $\left\{g_{t} \in \operatorname{Diff}(Y), t \in[0,1]\right\}$ such that $g_{0}=i d,\left.g_{t}\right|_{X}=\left.f_{t}\right|_{X}$ for every $t \in[0,1]$ and supp $\left\{g_{t}\right\}$ lies in $A$.

The details of the construction.

Denote by $O(0,0)$ the origin of coordinates on the plane $\mathbb{R}^{2}$. For every $r>0$ let $B_{r}=$ $=\left\{(x, y) \in \mathbb{R}^{2}: x^{2}+y^{2}<r^{2}\right\}$. Denote by $g: \mathbb{R}^{2} \rightarrow \mathbb{R}^{2}$ the diffeomorphism defined by $g(x, y)=(x / 2, y / 2)$.

Lemma 1. Let a diffeomorphism $\varphi_{0}: M^{2} \rightarrow M^{2}$ have a hyperbolic sink $\omega_{0}$ of period $m$ and let $\left(U_{0}, \psi_{0}\right)$ be a local chart of the manifold $M^{2}$ such that $\omega_{0} \in U_{0}, \psi_{0}\left(\omega_{0}\right)=O$ and $\varphi_{0}^{m}\left(U_{0}\right) \subset U_{0}$.

\footnotetext{
${ }^{1}$ The support supp $\left\{f_{t}\right\}$ of an isotopy $\left\{f_{t}\right\}$ is the closure of the set $\left\{x \in X: f_{t}(x) \neq f_{0}(x)\right.$ for some $t \in$
} $[0,1]\}$. 
Then there are neighborhoods $U_{1}, U_{2}$ of $\omega_{0}$ such that $U_{2} \subset U_{1} \subset U_{0}$ and there is a bifurcation free $\operatorname{arc} \varphi_{t}: M^{2} \rightarrow M^{2}, t \in[0,1]$ such that

1) the diffeomorphism $\varphi_{t}, t \in[0,1]$ coincides with $\varphi_{0}$ outside the set $\bigcup_{k=0}^{m-1} \varphi_{0}^{k}\left(U_{1}\right)$ and the set $\bigcup_{k=0}^{m-1} \varphi_{0}^{k}\left(\omega_{0}\right)$ is the hyperbolic sink orbit of period $m$ for every $\varphi_{t}$;

2) the diffeomorphism $\psi_{0} \varphi_{1}^{m} \psi_{0}^{-1}$ coincides with $g$ on the set $\psi_{0}\left(U_{2}\right)$.

Proof.

Let $\phi_{0}=\varphi_{0}^{m}$ and $\bar{\phi}_{0}=\psi_{0} \phi_{0} \psi_{0}^{-1}: \mathbb{R}^{2} \rightarrow \mathbb{R}^{2}$. To prove the lemma it suffices to construct neighborhoods $\bar{U}_{1}, \bar{U}_{2}$ of $O$ such that $\bar{U}_{2} \subset \bar{U}_{1} \subset \mathbb{R}^{2}$ and a bifurcation free $\operatorname{arc} \bar{\phi}_{t}: \mathbb{R}^{2} \rightarrow \mathbb{R}^{2}, t \in$ $[0,1]$ such that

$\left.1^{\prime}\right)$ the diffeomorphism $\bar{\phi}_{t}, t \in[0,1]$ coincides with $\bar{\phi}_{0}$ outside the set $\bar{U}_{1}$ and $O$ is the hyperbolic sink for all diffeomorphisms $\bar{\phi}_{t}$;

$\left.2^{\prime}\right)$ the diffeomorphism $\bar{\phi}_{1}$ coincides with $g$ on the set $\bar{U}_{2}$.

Indeed, one can get the arc $\varphi_{t}: M^{2} \rightarrow M^{2}$ from the arc $\bar{\phi}_{t}$ in the following way. Let $U_{1}=$ $=\psi_{0}^{-1}\left(\bar{U}_{1}\right), U_{2}=\psi_{0}^{-1}\left(\bar{U}_{2}\right)$ and $\phi_{t}=\psi_{0}^{-1} \bar{\phi}_{t} \psi_{0}$ on $U_{1}$. Then for every $t \in[0,1] \varphi_{t}$ coincides with $\varphi_{0}$ outside $\bigcup_{k=0}^{m-1} \varphi_{0}^{k}\left(U_{1}\right), \varphi_{t}(z)=\varphi_{0}(z)$ for $z \in \varphi_{0}^{k}\left(U_{1}\right), k \in\{0, \ldots, m-2\}$ and $\varphi_{t}(z)=\phi_{t}\left(\varphi_{0}^{1-m}(z)\right)$ for $z \in \varphi_{0}^{m-1}\left(U_{1}\right)$.

Now we construct the arc $\bar{\phi}_{t}$.

Denote by $Q: \mathbb{R}^{2} \rightarrow \mathbb{R}^{2}$ the differential of the map $\bar{\phi}_{0}$ at the point $O$. Since $O$ is a hyperbolic sink of $\bar{\phi}_{0}$, there exists a number $0<\lambda<1$ such that for every $v \in \mathbb{R}^{2}$

$$
\|Q(v)\| \leqslant \lambda\|v\|
$$

where $\|\cdot\|$ is the Lyapunov norm in $\mathbb{R}^{2}$ (see, for example, [9]). For every $r>0$ let $\tilde{B}_{r}=$ $=\left\{v \in \mathbb{R}^{2}:\|v\|<r\right\}$. Since $\bar{\phi}_{0}$ is a diffeomorphism with the fixed point $O$ and since all norms in $\mathbb{R}^{2}$ are equivalent, there is $\varepsilon_{1}>0$ such that for every $v \in \tilde{B}_{\varepsilon_{1}}$ the equation $\bar{\phi}_{0}(v)=Q(v)+$ $+\|v\| a(v)$ holds, where $\lim _{\|v\| \rightarrow 0} a(v)=0$. Therefore, there exists $0<\varepsilon_{2}<\varepsilon_{1}$ such that for every nonzero $v \in \tilde{B}_{\varepsilon_{2}}$ the inequality $\left\|\bar{\phi}_{0}(v)\right\|<\|v\|$ is true. Let $\bar{U}_{1}=\tilde{B}_{\varepsilon_{2}}$. Since $g\left(c l \bar{U}_{1}\right) \subset \bar{U}_{1}$, the family of the maps $\chi_{t}: \bar{U}_{1} \rightarrow \mathbb{R}^{2}$ is well-defined on the set $\bar{U}_{1}$ by

$$
\chi_{t}=(1-t) \bar{\phi}_{0}+t g .
$$

By construction, $\left\|\chi_{t}(v)\right\|<\|v\|$ for every nonzero $v \in \bar{U}_{1}$. Notice that the origin of coordinates is a fixed point of $\chi_{t}, \chi_{t}\left(c l \bar{U}_{1}\right) \subset \bar{U}_{1}$ for every $t \in[0,1]$ and the isotopy $\xi_{t}=\bar{\phi}_{0}^{-1} \chi_{t}$ joins the identity map with $\bar{\phi}_{0}^{-1} g$. Let $\bar{U}_{2}=\bar{\phi}_{0}\left(\bar{U}_{1}\right)$ (Fig. 9). Then $\xi_{t}\left(c l \bar{U}_{2}\right) \subset \bar{U}_{1}$ for all $t \in[0,1]$. By Proposition 5 , there is an isotopy $\Xi_{t}: \mathbb{R}^{2} \rightarrow \mathbb{R}^{2}$ which coincides with $\xi_{t}$ on $\bar{U}_{2}$ and which is the identity outside $\bar{U}_{1}$. The desired arc is defined by

$$
\bar{\phi}_{t}=\bar{\phi}_{0} \Xi_{t}
$$



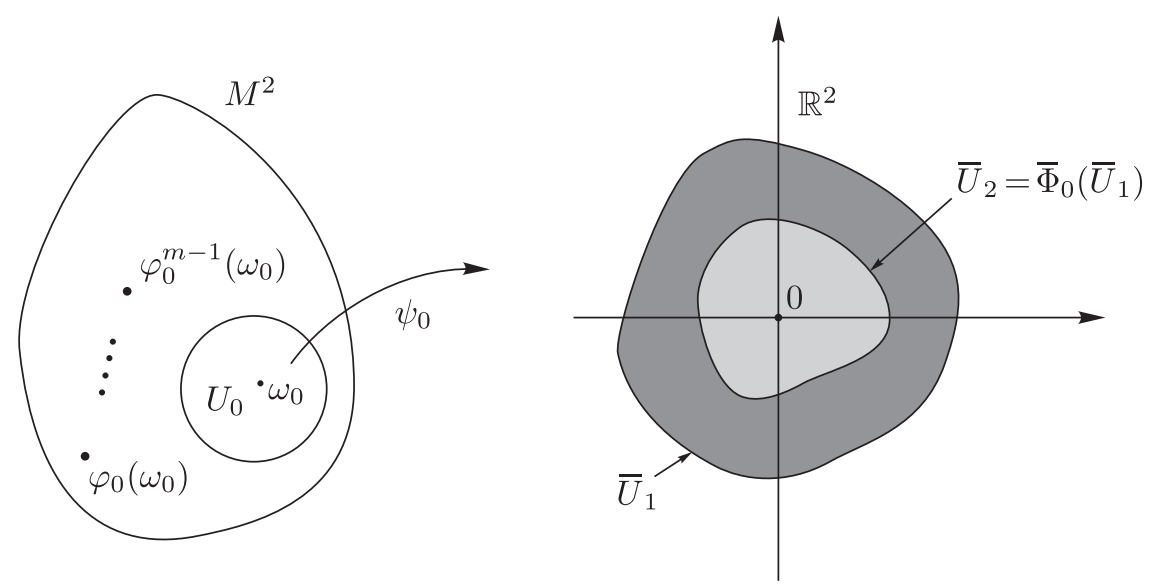

Fig. 9. Illustration for Lemma 1.

Lemma 2. Let a diffeomorphism $\varphi_{0}: M^{2} \rightarrow M^{2}$ have a hyperbolic sink $\omega_{0}$ and a hyperbolic saddle $\sigma_{0}$ such that the unstable separatrix $\gamma_{\varphi_{0}}$ of $\sigma_{0}$ lies in the basin of the sink $W_{\omega_{0}}^{s}$ and let $\sigma_{0}$ and $\omega_{0}$ be of the same period $m$. Let $\left(U_{0}, \psi_{0}\right)$ be a local chart of the manifold $M^{2}$ for which $\omega_{0} \in U_{0}, \psi_{0}\left(\omega_{0}\right)=O$ and $\varphi_{0}^{m}\left(U_{0}\right) \subset U_{0}$. Then there are neighborhoods $V_{1}, V_{2}$ of $\omega_{0}$ such that $V_{2} \subset V_{1} \subset U_{0}$ and there is a bifurcation free arc $\varphi_{t}: M^{2} \rightarrow M^{2}, t \in[0,1]$ with the following properties:

1) the diffeomorphism $\varphi_{t}, t \in[0,1]$ coincides with $\varphi_{0}$ outside the set $\bigcup_{k=0}^{m-1} \varphi_{0}^{k}\left(V_{1}\right)$ and the set $\bigcup_{k=0}^{m-1} \varphi_{0}^{k}\left(\omega_{0}\right)$ is the hyperbolic sink orbit of period $m$ for every $\varphi_{t}$;

2) $\psi_{0}\left(\gamma_{\varphi_{1}} \cap V_{2}\right) \subset O X_{1}$ where $\gamma_{\varphi_{1}}$ is the unstable separatrix of $\sigma_{0}$ with respect to $\varphi_{1}$ (Fig. 10).
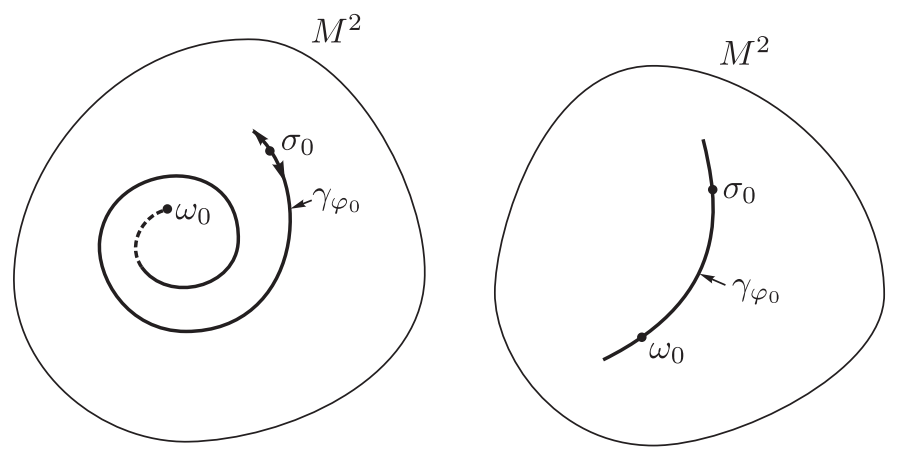

Fig. 10. Smoothing of the separatrix.

Proof.

Let $\phi_{0}=\varphi_{0}^{m}$ and $\bar{\phi}_{0}=\psi_{0} \phi_{0} \psi_{0}^{-1}: \mathbb{R}^{2} \rightarrow \mathbb{R}^{2}$. By Lemma 1, without loss of generality, suppose $\bar{\phi}_{0}=g$ on the disk $B_{2 r_{0}}$ for some $r_{0}>0$. Let $K_{0}=B_{2 r_{0}} \backslash B_{r_{0}}$ and $\gamma_{\bar{\phi}_{0}}=\psi_{0}\left(\gamma_{\varphi_{0}}\right)$. 
Denote by $E_{g}$ the set of contractions $\bar{\phi}: \mathbb{R}^{2} \rightarrow \mathbb{R}^{2}$ coinciding with $\bar{\phi}_{0}$ outside $B_{2 r_{0}}$ and coinciding with $g$ on $B_{r_{\bar{\phi}}}$ where $r_{\bar{\phi}} \leqslant 2 r_{0}$. For every $\bar{\phi} \in E_{g}$ let

$$
\gamma_{\bar{\phi}}=\bigcup_{k \in \mathbb{Z}} \bar{\phi}^{k}\left(\gamma_{\bar{\phi}_{0}} \cap K_{0}\right)
$$

By construction, the $\bar{\phi}$-invariant curve $\gamma_{\bar{\phi}}$ coincides with the $\bar{\phi}_{0}$-invariant curve $\gamma_{\bar{\phi}_{0}}$ outside the disk $B_{r_{0}}$. Then to prove the lemma it suffices to construct a curve of contractions $\bar{\phi}_{t}: \mathbb{R}^{2} \rightarrow$ $\mathbb{R}^{2}, t \in[0,1]$ such that

1') the diffeomorphism $\bar{\phi}_{t}, t \in[0,1]$ coincides with $\bar{\phi}_{0}$ outside the set $B_{r_{0}}$;

$\left.2^{\prime}\right)\left(\gamma_{\bar{\phi}_{1}} \cap B_{r_{\bar{\phi}_{1}}}\right) \subset O X_{1}$.

The $\operatorname{arc} \varphi_{t}: M^{2} \rightarrow M^{2}$ is constructed from the $\operatorname{arc} \bar{\phi}_{t}$ in the same way as in Lemma 1 if one sets $V_{1}=\psi_{0}^{-1}\left(B_{r_{0}}\right)$ and $V_{2}=\psi_{0}^{-1}\left(B_{r_{\bar{\phi}_{1}}}\right)$.

In order to construct the arc $\bar{\phi}_{t}$, we now introduce the following notations for an arbitrary diffeomorphism $\bar{\phi} \in E_{g}$.

Represent the 2-torus $\mathbb{T}^{2}$ as the orbit space of the diffeomorphism $g$ on the set $\mathbb{R}^{2} \backslash O$ and denote by $p: \mathbb{R}^{2} \backslash O \rightarrow \mathbb{T}^{2}$ the natural projection. Let $\hat{a}=p\left(O X_{1}\right)$ and $\hat{b}=p\left(\mathbb{S}^{1}\right)$ be generators of $\mathbb{T}^{2}$, let $K_{\bar{\phi}}=B_{r_{\bar{\phi}}} \backslash B_{r_{\bar{\phi}}} / 2, \hat{\gamma}_{\bar{\phi}}=p\left(\gamma_{\bar{\phi}} \cap K_{\bar{\phi}}\right)$ and $\hat{\gamma}_{\bar{\phi}}=p\left(\gamma_{\bar{\phi}} \cap K_{\bar{\phi}}\right)$. Then the curve $\hat{\gamma}_{\bar{\phi}}$ is the knot on the torus $\mathbb{T}^{2}$ with coordinates $\left\langle 1,-n_{\bar{\phi}}>, n_{\bar{\phi}} \in \mathbb{Z}\right.$ in the basis $\hat{a}, \hat{b}$ (see, for example, [5]).

The $\operatorname{arc} \bar{\phi}_{t}$ is the smooth product of the $\operatorname{arcs} \eta_{t}$ and $\zeta_{t}$ where

I) the arc $\eta_{t}, t \in[0,1]$ consists of contractions, it coincides with $\bar{\phi}_{0}$ outside the set $B_{r_{0}}$ and it joins the diffeomorphism $\eta_{0}=\bar{\phi}_{0}$ with some diffeomorphism $\eta_{1} \in E_{g}$ such that the knot $\hat{\gamma}_{\eta_{1}}$ has coordinates $<1,0>$ in the basis $\hat{a}, \hat{b}$;

II) the $\operatorname{arc} \zeta_{t} \in E_{g}, t \in[0,1]$ joins the diffeomorphism $\zeta_{0}=\eta_{1}$ to the diffeomorphism $\zeta_{1}$ such that $\hat{\gamma}_{\zeta_{1}}=\hat{a}$.
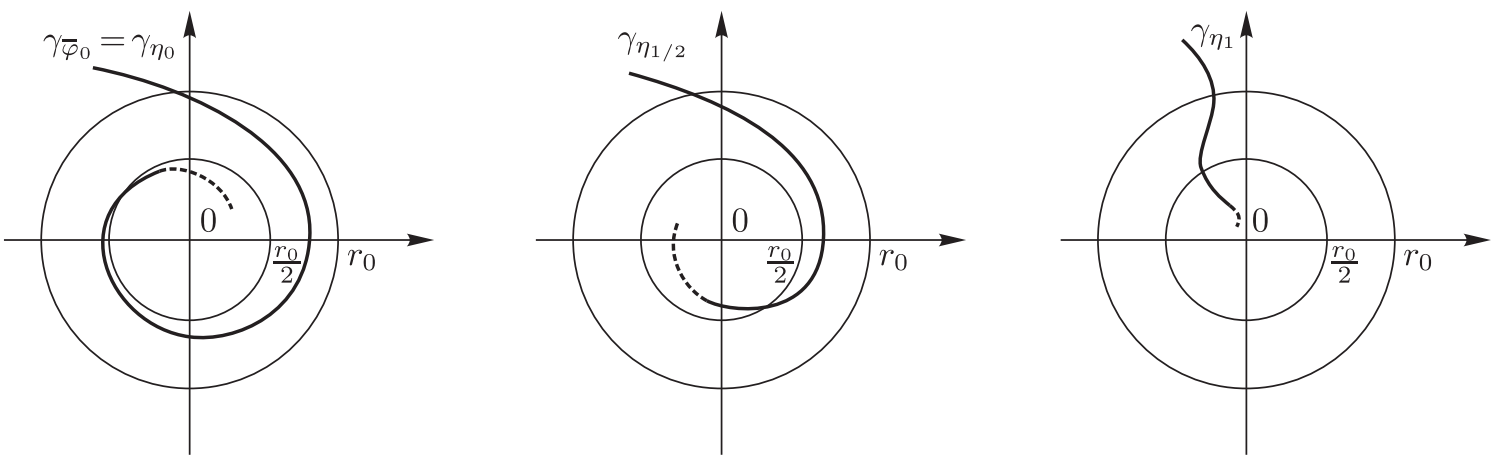

Fig. 11. Illustration to Lemma 2, Part I.

I) If $n_{\bar{\phi}}=0$, then let $\eta_{t}=\bar{\phi}_{0}$ for every $t \in[0,1]$. Otherwise define the diffeomorphism $\theta_{t}, t \in[0,1]: \mathbb{R}^{2} \rightarrow \mathbb{R}^{2}$ in such a way that $\theta_{t}(O)=O$ and

$$
\theta_{t}\left(\rho e^{i \varphi}\right)=\left\{\begin{array}{l}
\rho e^{i \varphi}, \rho>r_{0} \\
\rho e^{i\left(\varphi+4 n_{\bar{\phi}} \pi t\left(1-\frac{\rho}{r_{0}}\right)\right)}, \frac{r_{0}}{2} \leqslant \rho \leqslant r_{0} \\
\rho e^{i\left(\varphi+2 n_{\bar{\phi}} \pi t\right)}, \rho<\frac{r_{0}}{2} .
\end{array}\right.
$$


Then $\eta_{t}=\theta_{t} \bar{\phi}_{0}: \mathbb{R}^{2} \rightarrow \mathbb{R}^{2}$ is the desired arc (Fig. 11).

II) By construction, the diffeomorphism $\eta_{1} \in E_{g}$ and the knot $\hat{\gamma}_{\eta_{1}}$ has the coordinates $\langle 1,0\rangle$ in the basis $\hat{a}, \hat{b}$. According to [10], there is a diffeomorphism $\hat{h}: \mathbb{T}^{2} \rightarrow \mathbb{T}^{2}$ which is smoothly isotopic to the identity and such that $\hat{h}\left(\hat{\gamma}_{\eta_{1}}\right)=\hat{a}$. For $r>0$ let $K_{r}=B_{r} \backslash B_{r / 2}$. Pick an open cover $D=\left\{D_{1}, \ldots, D_{q}\right\}$ of the torus $\mathbb{T}^{2}$ such that the connected component $\bar{D}_{i}$ of the set $p^{-1}\left(D_{i}\right)$ is a subset of $K_{r_{i}}$ for some $r_{i}<\frac{r_{i-1}}{2}$ and $r_{1} \leqslant r_{0} / 2$. According to [11, Lemma de fragmentation] there are diffeomorphisms $\hat{w}_{1}, \ldots, \hat{w}_{q}: \mathbb{T}^{2} \rightarrow \mathbb{T}^{2}$ smoothly isotopic to the identity, with the following properties:

i) for each $i \in\{1, \ldots, q\}$ there exists a smooth isotopy $\left\{\hat{w}_{i, t}\right\}$ which is the identity outside $D_{i}$ and which joins the identity and $\hat{w}_{i}$;

ii) $\hat{h}=\hat{w}_{1} \ldots \hat{w}_{q}$.

Let $w_{i, t}: \mathbb{R}^{2} \rightarrow \mathbb{R}^{2}$ be a diffeomorphism which coincides with $\left(\left.p\right|_{K_{r_{i}}}\right)^{-1} \hat{w}_{i, t} p$ on $K_{r_{i}}$ and which coincides with the identity outside $K_{r_{i}}$ (Fig. 12). Then the desired arc is defined by

$$
\zeta_{t}=\nu_{1} w_{1, t} \ldots w_{q, t}
$$
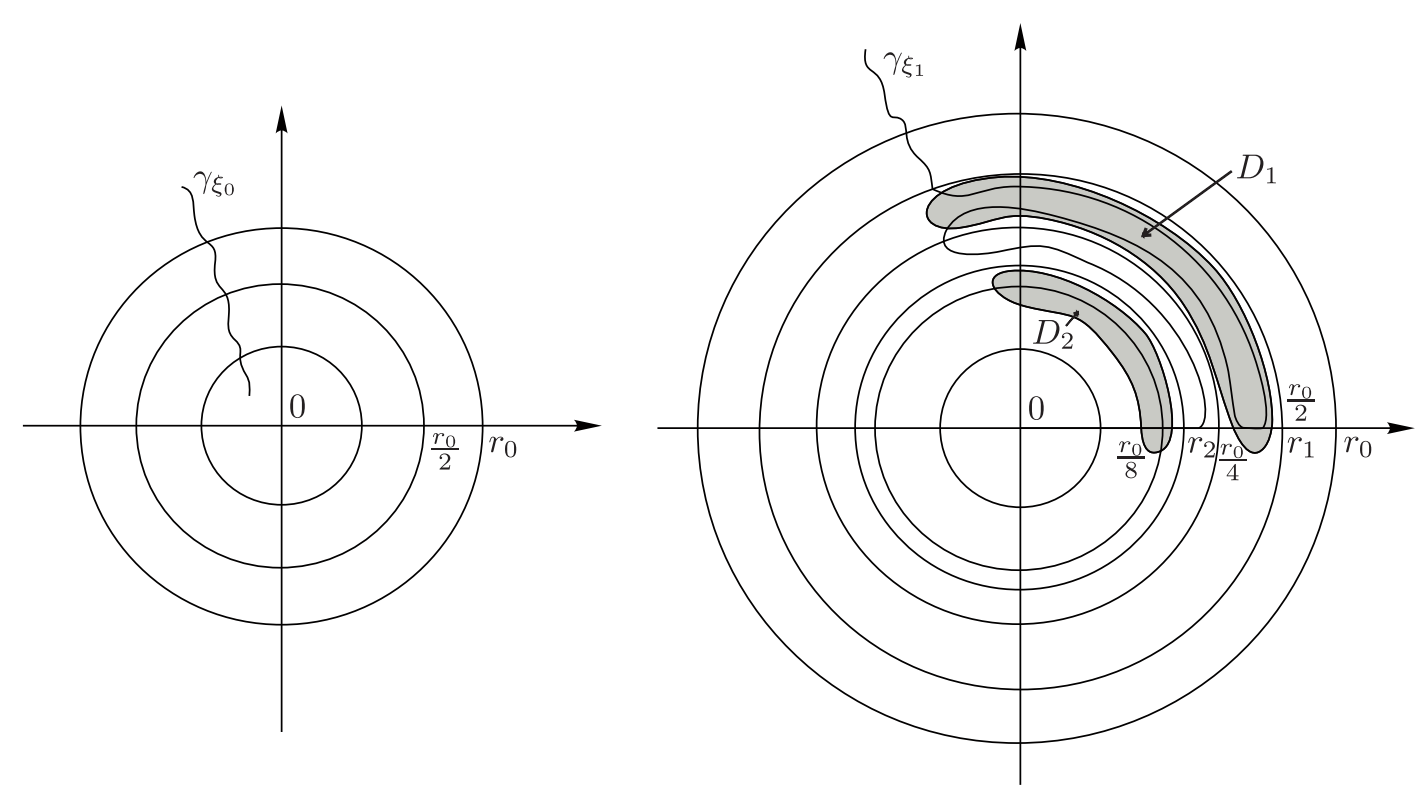

Fig. 12. Illustration to Lemma 2, Part II.

\section{Proof of Theorem 2}

In this section we construct a stable arc that joins the diffeomorphism $f \in G$ and "sourcesink" diffeomorphism on the 2-sphere. The key technical tool to do this is the following lemma.

Lemma 3. Let the nonwandering set of a gradient-like diffeomorphism $\varphi: M^{2} \rightarrow M^{2}$ contain a fixed saddle $\sigma$ of negative orientation type and such that $\operatorname{cl}\left(W_{\sigma}^{u}\right)=W_{\sigma}^{u} \cup \omega \cup \varphi(\omega)$, 
where $\omega$ is a sink of period two. Then there exists a stable arc $H_{\varphi, \varphi_{\sigma}^{u}, t}$ joining $\varphi$ to a gradientlike diffeomorphism $\varphi_{\sigma}^{u}$ whose nonwandering set is the same as $\Omega_{f}$ but with the points $\sigma, \omega, f(\omega)$ removed.

Then the desired arc is:

1) $H_{f_{1},\left(f_{1}\right)_{\sigma}^{u}, t}$ for $f_{1} \in G_{1}$;

2) $H_{f_{2},\left(f_{2}\right)_{\sigma_{1}}^{u}, t} * H_{\psi_{\sigma_{2}}^{u}, t}^{-1}$ where $\psi=\left(\left(f_{2}\right)_{\sigma_{1}}^{u}\right)^{-1}$ for $f_{2} \in G_{2}$;

3) $H_{f_{3}^{-1},\left(f_{3}^{-1}\right)_{\sigma}^{u}, t}$ for $f_{3} \in G_{3}$.

Proof of Lemma 3.

From Lemmas 1 and 2 it follows that there exists a local chart $(U, \psi)$ of the manifold $M^{2}$ such that $\omega \in U, \psi(\omega)=O, f^{2}(U) \subset U$ and $\psi(\gamma \cap U) \subset O X_{1}$. Let $A_{f}=W_{\sigma}^{u} \cup \omega \cup f(\omega)$ and $l=W_{\sigma}^{u} \cup \psi^{-1}\left(O X_{1}\right) \cup f\left(\psi^{-1}\left(O X_{1}\right)\right)$. Then $l$ is a smooth curve which contains $A_{f}$, for which the points $\omega, f(\omega)$ are interior and $f(l) \subset l$. Let $\Pi_{1}=\left\{\left(x_{1}, x_{2}\right) \in \mathbb{R}^{2}:\left|x_{i}\right| \leqslant \frac{1}{2}\right\}$. Define the diffeomorphism $\tilde{\varphi}: \Pi_{1} \rightarrow \mathbb{R}^{2}$ by

$$
\tilde{\varphi}\left(x_{1}, x_{2}\right)=\left(-\frac{11}{10} x_{1}+x_{1}^{3},-\frac{x_{2}}{2}\right) .
$$

By construction, $\tilde{\varphi}\left(\Pi_{1}\right) \subset \operatorname{int} \Pi_{1}$. The diffeomorphism $\tilde{\varphi}$ has the saddle $O$ and the periodic sink orbit $\left\{P_{0}, \tilde{\varphi}\left(P_{0}\right)\right\}$ where $P_{0}\left(-x_{0}, 0\right), \tilde{\varphi}\left(P_{0}\right)=\left(x_{0}, 0\right), x_{0} \in(0,1 / 2)$. Let $\Pi_{2}=\tilde{\varphi}\left(\Pi_{1}\right)$. Pick a closed neighborhood $V$ of the set $A_{f}$ and pick a diffeomorphism $\beta: V \rightarrow \Pi_{1}$ such that $f(V) \subset$ int $V, \beta(l \cap V)=O x_{1} \cap \Pi_{1}, \beta(f(V))=\Pi_{2}, \beta(\omega)=P_{0}$ and $\beta(f(\omega))=\tilde{\varphi}\left(P_{0}\right)$ (Fig. 13). Let $\tilde{f}=\beta f \beta^{-1}: \Pi_{1} \rightarrow \Pi_{2}$. Then the family of maps $\chi_{t}: \Pi_{2} \rightarrow \mathbb{R}^{2}$ is well-defined on the set $\Pi_{2}$ by

$$
\chi_{t}=(1-t) \tilde{f}+t \tilde{\varphi} .
$$
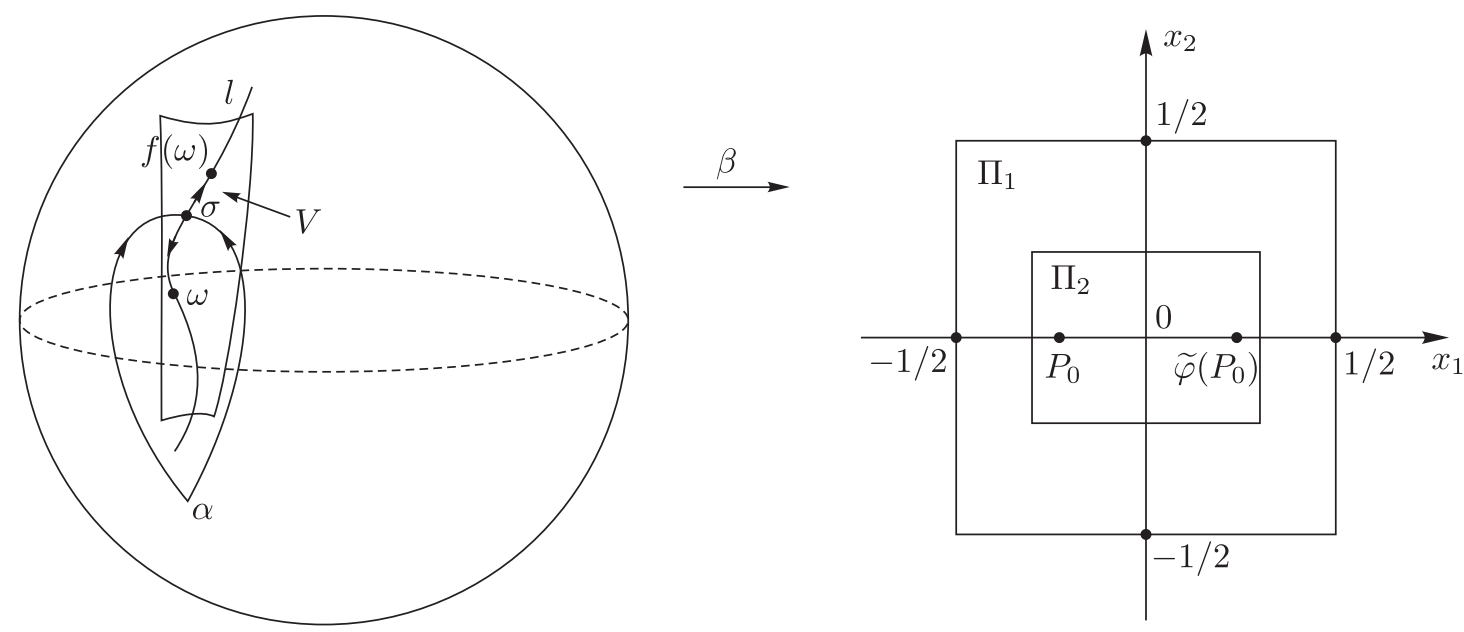

Fig. 13. Illustration to Lemma 3.

By construction, $\chi_{t}\left(\Pi_{2}\right) \subset$ int $\Pi_{2}$ for every $t \in[0,1]$. Notice that the origin of coordinates is the fixed saddle for the diffeomorphism $\chi_{t}$ and the points $P_{0}, \tilde{\varphi}\left(P_{0}\right)$ form the sink orbit. The isotopy $\xi_{t}=\left.\tilde{f}^{-1} \chi_{t}\right|_{\Pi_{2}}$ joins the identity map and the diffeomorphism $\tilde{f}^{-1} \tilde{\varphi}$ and $\xi_{t}\left(\Pi_{2}\right) \subset$ int $\Pi_{1}$. 
By Proposition 5, there is an isotopy $\Xi_{t}: \Pi_{1} \rightarrow \Pi_{1}$ which coincides with $\xi_{t}$ on $\Pi_{2}$ and which is the identity on $\partial \Pi_{1}$. Let

$$
\tilde{h}_{t}=\tilde{f} \Xi_{t} .
$$

Notice that $\tilde{h}_{1}=\tilde{\varphi}$ on $\Pi_{2}$. Let $\Pi_{3}=\tilde{\varphi}\left(\Pi_{2}\right)$. Define the arc $\eta_{t}: \Pi_{3} \rightarrow \mathbb{R}^{2}$ by

$$
\eta_{t}\left(x_{1}, x_{2}\right)=\left(-x_{1}\left(1+\frac{1}{10}(1-2 t)\right)+x_{1}^{3},-\frac{x_{2}}{2}\right) .
$$

By construction, $\eta_{t}\left(\Pi_{3}\right) \subset$ int $\Pi_{3}$ for every $t \in[0,1]$ and the isotopy $\zeta_{t}=\tilde{\varphi}^{-1} \eta_{t}$ joins the identity map with the diffeomorphism $\tilde{\varphi}^{-1} \eta_{1}$ and $\zeta_{t}\left(\Pi_{3}\right) \subset$ int $\Pi_{2}$. By Proposition 5 , there is an isotopy $\theta_{t}: \mathbb{R}^{2} \rightarrow \mathbb{R}^{2}$, which coincides with $\zeta_{t}$ on $\Pi_{3}$ and which is the identity outside $\Pi_{2}$. Let

$$
\tilde{\Theta}_{t}=\tilde{\varphi} \theta_{t} .
$$

Then the desired $\operatorname{arc} H_{\varphi, \varphi_{\sigma}^{u}, t}$ is the product of the $\operatorname{arcs} h_{t}, \Theta_{t}: M^{2} \rightarrow M^{2}$ where $h_{t}$ coincides with $f$ outside $V, h_{t}(z)=\beta^{-1}\left(\tilde{h}_{t}(\beta(z))\right)$ for $z \in V$ and $\beta_{t}$ coincides with $h_{1}$ outside $h_{1}(V)$, $\Theta_{t}(z)=\beta^{-1}\left(\tilde{\Theta}_{t}(\beta(z))\right)$ for $z \in h_{1}(V)$.

\section{References}

[1] Newhouse, S., Palis, J., and Takens, F., Stable Arcs of Diffeomorphisms, Bull. Amer. Math. Soc., 1976, vol. 82, no. 3, pp. 499-502.

[2] Newhouse, S. and Peixoto, M. M., There Is a Simple Arc Joining Any Two Morse-Smale Flows, in Trois études en dynamique qualitative, Astérisque, vol. 31, Paris: Soc. Math. France, 1976, pp. 15-41.

[3] Nozdrinova, E. V., Rotation Number As a Complete Topological Invariant of a Simple Isotopic Class of Rough Transformations of a Circle, Russian J. Nonlinear Dyn., 2018, vol. 14, no. 4, pp. 543-551.

[4] Blanchard, P. R., Invariants of the NPT Isotopy Classes of Morse-Smale Diffeomorphisms of Surfaces, Duke Math. J., 1980, vol.47, no. 1, pp. 33-46.

[5] Grines, V., Medvedev, T., and Pochinka, O., Dynamical Systems on 2- and 3-Manifolds, Dev. Math., vol. 46, New York: Springer, 2016.

[6] von Kerékjártó, B., Über die periodischen Transformationen der Kreisscheibe und der Kugelflache, Math. Ann., 1919, vol. 80, no. 1, pp. 36-38.

[7] Newhouse, S., Palis, J., and Takens, F., Bifurcations and Stability of Families of Diffeomorphisms, Inst. Hautes Études Sci. Publ. Math., 1983, No.57, pp. 5-71.

[8] Milnor, J., Lectures on the h-Cobordism Theorem, Princeton, N.J.: Princeton Univ. Press, 1965.

[9] Katok, A. and Hasselblatt, B., Introduction to the Modern Theory of Dynamical Systems, Encyclopedia Math. Appl., vol. 54, Cambridge: Cambridge Univ. Press, 1995.

[10] Rolfsen, D., Knots and Links, Math. Lect. Ser., No. 7, Berkeley, Calif.: Publish or Perish, Inc., 1976.

[11] Banyaga, A., On the Structure of the Group of Equivariant Diffeomorphisms, Topology, 1977, vol. 16, no. 3, pp. 279-283. 